ices and personnel (Box I). There is, in particular, a dearth of trained professionals to treat patients, which forces everyone to seek novel solutions.

"Health care workers have to be innovative when they have so few resources," says Apollo Dalton Nyangasi, chairman of the Uganda Medical Workers Union.

Most doctors are trained at Kampala's Makerere University, I of 3 medical schools in the country. Students at Makerere attend 5 years of classes, followed by I year of full-time work at $\mathrm{Mu}$ lago Hospital. During that latter year, they are expected to toil 7 days a week for 12 months, all unpaid, which makes it extremely difficult for children from poor families to attend medical school.

Remarkably, despite the obstacles, funding shortfalls and challenges, there are success stories.

In the north, where rebels waged a 20-year civil war against the Ugandan government until peace was negotiated last year, immunization rates and overall health education among the population is very high.

This is mostly because many of the north's 4.5 million people lived in displaced persons' camps, some for parts or all of the 2 decade duration of the conflict. As such, international organizations had a centralized, captive audience to treat and educate on health concerns.

As people begin moving home, the Ugandan government has given $25 \%$ more funding to northern districts to help the region's health care system adjust to the changing environment.

Uganda is also often held up as an HIV/AIDS success story.

Having been $\mathrm{I}$ of the first countries to face an AIDS epidemic, it was also I of the first to respond with a coordinated campaign of education and testing.

Numbers vary, but it is generally held that Uganda's HIV/AIDS infections peaked in 199I, when about $15 \%$ of the population was infected. Today, less than $7 \%$ is said to be infected and there continue to be large-scale campaigns encouraging abstinence, condom use and faithfulness in marriage as ways to bring the number further down. Christopher Mason, Kampala, Uganda

DOI:Io.I503/cmaj.071795

\section{Mapping infectious}

\section{diseases in Africa}

M athematical modeling, by predicting the trajectory of infection, can become the next tool experts use to tackle infectious diseases that persist despite decades of effort to contain them, according to Canadian and African researchers attending a recent mathematical epidemiology conference in Kampala, Uganda.

The researchers hope their joint efforts to bridge mathematics and science will yield useful models that help policy-makers decide when and how to deploy resources (Figure I).

"Many diseases, such as malaria, have cyclical behaviour," explains Arvind Gupta, scientific director at British Columbia-based Mathematics of Information Technology and Complex Systems, which organized the meeting. "But often resources are needed at their most when malaria rates are at their lowest."

Often, when an infectious disease is most prevalent, it also has its lowest rate of new infections because the affected population is educated about the disease and resources are deployed. "It's when a disease dips that resources disappear and complacency sets in," Gupta says.

Modelling, or mathematical epidemi- ology, is meant to combat that tendency by showing policy-makers the correlation between infection rates, resource allocation and a disease's behaviour.

By doing that, researchers hope to avoid the wild fluctuations that often persist in infection rates, and instead focus on keeping the "R" value (rate of infection) at less than I at all times, meaning less than I person is infected with the disease for every I person who has it. If that can be accomplished, the disease begins a slow but steady march towards being eliminated.

Conference participants, who say that policy-makers do not fully recognize the role mathematical models can play in disease prevention, were optimistic that the groundwork has been laid for future collaborations that tap Canadian expertise in mathematical epidemiology and first-hand African knowledge of disease patterns.

"There are very good scientists here doing work in a resource-poor setting," says Abba Gumel, director of the University of Manitoba's Institute of Industrial Mathematics. "They don't have access to the resources we do in Canada. But we see that as a chance to build some real collaborations here because these are scientists who know the lay of the land and who to work with." Christopher Mason, Kampala, Uganda

DOI:I0.1503/cmaj.071749

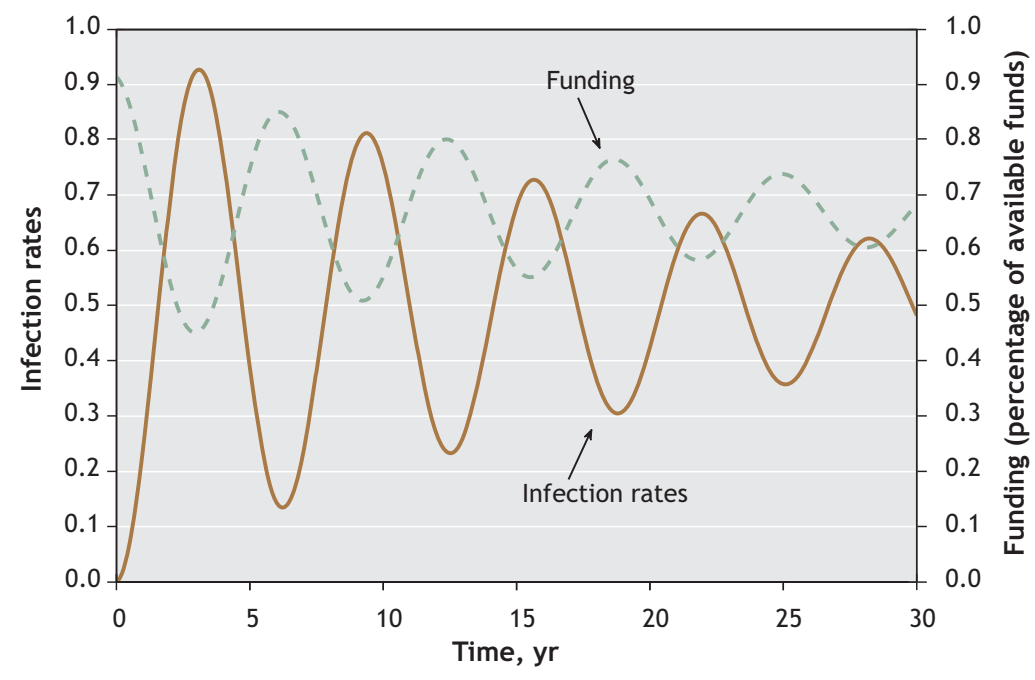

Figure 1: Researchers say that infectious diseases can be curbed through using of mathematical models demonstrating the correlation between infection rates, resource allocation and a disease's behaviours. 